\title{
A NOTE ON THE INDEPENDENCE AND TOTAL DEPENDENCE OF MAX I.D. DISTRIBUTIONS
}

\author{
J. HÜSLER, ${ }^{*}$ University of Bern
}

\begin{abstract}
We show that the simple characterizations given by Takahashi for the independence and the total dependence of a multivariate extreme value distribution do not hold for the larger class of maximum infinitely divisible ( $\max$ i.d.) distributions. This holds also for sup self-decomposable distributions.

INDEPENDENCE; TOTAL DEPENDENCE; MAX INFINITELY DIVISIBLE; SUP SELF-DECOMPOSABLE; MAX STABLE; EXTREME VALUE DISTRIBUTION
\end{abstract}

Consider a random vector $\boldsymbol{X} \in R^{d}$ with multivariate extreme value distribution $G$ which is characterized by the property of the maximum stability. $G$ is max stable if for every $r>0$ there exist vectors $\alpha(r)$ and $\beta(r)$ such that $G^{r}(\alpha(r) x+\beta(r))=G(x)$. It is known that $G$ is associated (cf. Marshall and Olkin (1983)) and has therefore a positive dependence structure. The following inequality holds:

$$
\prod_{j=1}^{d} G_{j}\left(x_{j}\right) \leqq G(x) \leqq \min _{j}\left(G_{j}\left(x_{j}\right)\right)
$$

(cf. Galambos (1978)), where $G_{j}$ is the $j$ th univariate marginal of $G,(j \leqq d)$. In an application it is rather interesting to know whether $G$ has independent components which gives the lower bound of (1), or whether $G$ has totally dependent components giving the upper bound of (1).

Takahashi (1987), (1988) proved recently that the following surprisingly simple conditions are necessary and sufficient for the independence and the total dependence, respectively.

The condition for independence is the following one: $G(\boldsymbol{x})=\prod_{j=1}^{d} G j\left(x_{j}\right)$ iff there exists $z \in R^{d}$ such that for all $j<d$,

$$
G_{j}\left(z_{j}\right) \in(0,1) \text { and } G(z)=\prod_{j=1}^{d} G_{j}\left(z_{j}\right)
$$

In the same way we have the condition for the total dependence: $G(x)=\min _{j}\left(G_{j}\left(x_{j}\right)\right)$ iff there exists $z \in R^{d}$ such that for all $j \leqq d$,

$$
G_{j}\left(z_{j}\right)=G_{1}\left(z_{1}\right) \in(0,1) \text { and } G(z)=G_{1}\left(z_{1}\right) .
$$

A much larger class of distribution $G$ is used for the asymptotic limit of extreme values of sequence of non-identically distributed random vectors (cf. Hüsler (1988a,b)). We showed that such limit laws $G$ are max i.d. which means that for any $t>0$ there exists a multivariate distribution $G_{t}$ on $R^{d}$ such that $G=G_{t}^{t}$ (cf. Balkema and Resnick (1977) or Resnick (1987)). In this note we show that the above simple conditions (2) and (3) are not sufficient to guarantee that a general max i.d. (maximum infinitely divisible) distribution is independent and totally dependent, respectively. This is proved by the following simple examples for the case $d=2$, which can be easily generalized for any $d>2$.

\footnotetext{
Received 26 August 1988; revision received 5 December 1988.

* Postal address: Department of Math. Statistics, University of Bern, Sidlerstr. 5, CH-3012 Bern, Switzerland.
} 
1. Let $d=2, \boldsymbol{Y} \sim F_{Y}(x, y)=x y$, an independent uniform random vector on $[0,1]^{2}$, and $\boldsymbol{X} \sim F_{\boldsymbol{X}}(x, y)=\min (x, y)$ a totally dependent random vector on $[0,1]^{2}$. Let $\boldsymbol{X}$ and $\boldsymbol{Y}$ be independent random vectors. Obviously they are max i.d.

Define $\left.\boldsymbol{Z}=\max \left(\boldsymbol{X}-\frac{1}{2}\right) \boldsymbol{1}, \boldsymbol{Y}\right) \sim G(x, y)$, where all algebraic operations are taken componentwise, with $1=(1,1)$. Since the function used $(x, y)-\left(\frac{1}{2}, \frac{1}{2}\right)$ and the maximum function are non-decreasing, $\boldsymbol{Z}$ is also max i.d. (cf. Resnick (1987)).

Note that $G(x, y)=\min \left(x+\frac{1}{2}, y+\frac{1}{2}, 1\right) x y$ with $G_{1}(x)=\min \left(x+\frac{1}{2}, 1\right) x$ and $G_{2}(y)=$ $\min \left(y+\frac{1}{2}, 1\right) y$; thus it shows that for every $(x, y) \nless\left(\frac{1}{2}, \frac{1}{2}\right)$ we get $G(x, y)=x y=G_{1}(x) G_{2}(y)$, which does not hold for $(x, y)<\left(\frac{1}{2}, \frac{1}{2}\right)$. This example demonstrates that $(2)$ is not sufficient for the independence of a max i.d. distribution.

2. Let $d=2$ and $\boldsymbol{X}, \boldsymbol{Y}$ be as in the first example. Define now $\boldsymbol{Z}=\max \left(\boldsymbol{X}, \boldsymbol{Y}-\left(\frac{1}{2}\right) \boldsymbol{1}\right)$ which is again a max i.d. random vector. The distribution of $Z$ is $G(x, y)=\min (x, y) \min (x+$ $\left.\frac{1}{2}, 1\right) \min \left(y+\frac{1}{2}, 1\right)$ with $G_{1}(x)=x \min \left(x+\frac{1}{2}, 1\right)$ and $G_{2}(y)=y \min \left(y+\frac{1}{2}, 1\right)$. For $(x, y) \nless$ $\left(\frac{1}{2}, \frac{1}{2}\right)$ we get $G(x, y)=\min \left(G_{1}(x), G_{2}(y)\right)$, which does not hold for $(x, y)<\left(\frac{1}{2}, \frac{1}{2}\right)$. This example shows that (3) is not sufficient for the total dependence of a max i.d. distribution.

It is also easily seen by using the so-called exponent measure $\mu_{1}$ that the distribution of the first example cannot be independent. By using $\mu_{2}$ it it easily seen that the second distribution cannot be totally dependent. In the first case $\mu_{1}$ should have the mass on the two axes through $(0,0)$ and in the second case $\mu_{2}$ should have its mass only on the line $t 1$ (the diagonal of $\left.[0,1]^{2}\right)$ (cf. Resnick (1987)).

A subclass of the max i.d. distribution is dealt with by Gerritse (1986). An r.v. $\boldsymbol{X}$ is called sup self-decomposable if for every $t>0$ there exists an r.v. $\boldsymbol{X}_{t}$, independent of $\boldsymbol{X}$, such that $\boldsymbol{X} \stackrel{\mathrm{d}}{=} \max \left((\boldsymbol{X}-t 1), X_{t}\right)$. It is easily seen that the two r.v.'s $\boldsymbol{X}$ and $\boldsymbol{Y}$ of our examples are also sup self-decomposable. Since also the maximum of sup self-decomposable r.v.'s is again sup self-decomposable, and in the same way a translated version of such a r.v., we see immediately that in both examples $Z$ is also sup self-decomposable. Thus the conditions of Takahashi are also not sufficent in the class of sup self-decomposable r.v.'s.

\section{Acknowledgement}

This paper was written while the author was a Visiting Fellow of the ANU, Canberra.

\section{References}

[1] Balkema, A. A. and Resnick, S. I. (1977) Max-infinite divisibility. J. Appl. Prob. 14, 309-313.

[2] Galambos, J. (1978) The Asymptotic Theory of Extreme Order Statistics. Wiley, New York. (2nd edn (1987) Krieger, Florida).

[3] Gerritse, G. (1986) Supremum self-decomposable random vectors. Prob. Theory. Rel. Fields 72, 17-33.

[4] HüsLER, J. (1988a) Limit properties for multivariate extreme values in sequences of independent, non-identically distributed random vectors. Stoch. Proc. Appl. to appear.

[5] HüsLER, J. (1988b) Limit distributions of multivariate extreme values in nonstationary sequences of random vectors. To be published.

[6] Marshall, A. W. AND Olkin, I. (1983) Domains of attraction of multivariate extreme value distributions. Ann. Prob. 11, 168-177.

[7] RESNICK, S. I. (1987) Extreme Values, Regular Variation, and Point Processes. Springer-Verlag, Berlin.

[8] TAKAHASHI, R. (1987) Some properties of multivariate extreme value distributions and multivariate tail equivalence. Ann. Inst. Statist. Math. 39, A, 637-647.

[9] TAKahashi, R. (1988) Characterizations of a multivariate extreme value distribution. Adv. Appl. Prob. 20, 235-236. 